\title{
Nueva especie de Brachistosternus Pocock (Scorpiones: Bothriuridae) del sur del Perú
}

\section{A new species of Brachistosternus Pocock (Scorpiones: Bothriuridae) from southern Peru}

\author{
José Antonio Ochoa*
}

\begin{abstract}
Resumen
Se describe Brachistosternus turpuq, nueva especie de escorpión Bothriuridae del desierto costero en el sur del Perú, colectada en los cerros alrededores de Mejía (Departamento de Arequipa). Esta especie pertenece al subgénero Brachistosternus (Leptosternus) Maury, y está muy relacionada con las especies argentinas $B$. pentheri Mello-Leitão, $B$. multidentatus Maury y $B$. angustimanus Ojanguren Affilastro \& Roig Alsina. La nueva especie se diferencia de ellas por el menor número de setas en el segmento caudal $\mathrm{V}$ y del tarso III, algunos detalles en el hemiespermatóforo (espinas del lóbulo interno) y en el patrón de pigmentación del prosoma y del segmento caudal $\mathrm{V}$.
\end{abstract}

Palabras clave: Scorpiones, Bothriuridae, Brachistosternus, Perú, Desierto costero.

\section{Abstract}

Brachistosternus turpuq, new bothriurid species from the coastal desert of southern Peru is described. It was collected in hills around Mejía (departamento Arequipa). This species belongs to the subgenus Brachistosternus (Leptosternus) Maury, and is related to the Argentinean species $B$. pentheri Mello-Leitão, B. multidentatus Maury and B. angustimanus Ojanguren Affilastro \& Roig Alsina. The new species differs from these species by a lower count of setae on the metasomal segment $V$ and tarsus III, some details of the hemispermatophore (spines of the internal lobe), and the pigmentation pattern of the prosoma and metasomal segment $\mathrm{V}$.

Key words: Scorpiones, Bothriuridae, Brachistosternus, Perú, Coastal desert.

\section{Introducción}

El género Brachistosternus Pocock, 1893 comprende escorpiones andino-patagónicos que se distribuyen desde Ecuador hasta Argentina, entre el nivel de mar y más de 4000 metros de altitud (Maury, 1973; Acosta y Maury, 1998; Lowe y Fet, 2000). El grupo incluye actualmente 24 especies nominales las que están incluidas en tres subgéneros: Brachistosternus (Brachistosternus) Pocock, con una especie: $B$. (B.) ehrenbergii (Gervais, 1841); Brachistosternus

\footnotetext{
* Becario Secyt. Cátedra de Diversidad Animal I, Facultad de Ciencias Exactas, Físicas y Naturales, Universidad Nacional de Córdoba, Av. Vélez Sarsfield 299, 5000 Córdoba, Argentina. Email:jochoa@com.uncor.edu; jaochoac@hotmail.com
}

(Ministernus) Francke, 1985, con 3 especies y Brachistosternus (Leptosternus) Maury, 1973, con 17 especies y además tres especies de posición incierta (Maury, 1973; Lowe y Fet, 2000). Estos tres subgéneros fueron reconocidos por Maury (op. cit.), quien basó su clasificación principalmente en la disposición de las tricobotrias, pero sin duda que dicha subdivisión necesita una revisión más detallada que incluya un análisis cladístico del grupo que permita comprobar la monofilia de cada subgénero.

Para el Perú a la fecha se conocen con seguridad 4 especies de los 3 subgéneros: $B$. (B.) ehrenbergii; B. (M.) andinus Chamberlin, 1916; B. (L.) titicaca Ochoa \& Acosta, 2002 y $B$ (L.) quiscapata Ochoa \& 
Acosta, 2002 (Aguilar y Meneses, 1970; Francke, 1977; Ochoa y Acosta, 2002); una quinta especie, B. (M.) peruvianus Piza, 1974, es considerada como posible sinónimo de $B$. andinus (Maury, 1978; Lowe \& Fet, 2000). Otras tres especies, B. castroi Mello-Leitão, 1940; B. weijenberghii (Thorell, 1876) y $B$. holmbergi Carbonell, 1923 (citadas previamente para el país), fueron excluidas del elenco de la fauna de escorpiones de Perú (Ochoa y Acosta, 2002).

En el presente trabajo se describe una nueva especie perteneciente al subgénero $B$. (Leptosternus) y que está muy emparentada con las especies argentinas $B$. pentheri Me1lo-Leitão, 1931; B. multidentatus Maury, 1984 y B. angustimanus Ojanguren Affilastro \& RoigAlsina, 2001. Fue colectada a $340 \mathrm{msnm}$., en cerros cercanos a la localidad de Mejía (desierto costero del departamento de Arequipa), en un área que correspondería a la parte baja de las laderas donde se forma la vegetación de las "lomas" (Péfaur, 1981). Junto con esta especie se encontraron ejemplares de $B$. ehrenbergii.

\section{Abreviaturas}

CDA: Cátedra de Diversidad Animal I, Facultad de Ciencias Exactas, Físicas y Naturales, Universidad Nacional de Córdoba, Argentina.

MHNC: Museo de Historia Natural, Facultad de Ciencias Biológicas, Universidad Nacional de San Antonio Abad del Cusco, Perú.

MUSM: Museo de Historia Natural, Universidad Nacional Mayor de San Marcos, Lima, Perú.

Carenas de los segmentos caudales I-V:

$\mathrm{DL}=$ dorsal lateral,

LsM = lateral supramediana,

$\mathrm{LM}=$ lateral mediana,

LIM = lateral inframediana,

$\mathrm{V}_{\mathrm{L}}=$ ventral lateral,

VSM $=$ Ventral submediana,

$\mathrm{V}_{\mathrm{M}}=$ Ventral mediana.
Brachistosternus (Leptosternus) turpuq

n. sp.

(Figs. 1-14)

\section{Etimología}

El nombre específico deriva de la palabra Quechua turpuq que significa 'aguijoneador' y hace alusión a la peculiar agresividad que presentaron los ejemplares de esta especie al momento de ser capturados. Al ser derivado directamente de una lengua no latina, el término se considera indeclinable (Art. 31.2.3 del Código Internacional de Nomenclatura Zoológica, 4. ${ }^{a}$ ed., 1999).

\section{Serie Típica}

Holotipo macho (MUSM), Alotipo hembra (MUSM), 1 hembra paratipo (CDA 000,098), 1 macho, 1 hembra paratipos (MHNC), Perú: Cerros alrededores de Mejía (340 m; $17^{\circ} 05^{\text {' }}$ $\left.\mathrm{S}, 71^{\circ} 53^{\prime} \mathrm{W}\right)$, prov. Islay, departamento Arequipa (Fig. 15), 11 febrero 2000, U. Zanabria, H. Alayo \& J. A. Ochoa.

\section{Otros materiales estudiados (no tipos)}

PERÚ, Departamento Arequipa: Cerros alrededores de Mejía, Islay, 15 diciembre 1996 (H. Zeballos \& J. A. Ochoa), 1 hembra, 1 macho juv. (MHNC); igual localidad y datos que la serie típica, 1 hembra (MHNC).

\section{Diagnosis}

Especie perteneciente al subgénero Brachistosternus (Leptosternus). Por la forma del telson (alto, granuloso ventralmente y con aguijón largo), la forma de las glándulas caudales (alargadas y delgadas), pinzas de los pedipalpos (relativamente delgadas con los dedos proporcionalmente largos) y número elevado de dientes pectíneos, las especies nominales que más se aproximan a $B$. turpuq $\mathrm{n}$. sp. son: $B$. pentheri, $B$. multidentatus y $B$. angustimanus (las tres especies distribuidas en Argentina). La nueva entidad se distingue de dichas especies por el menor número de setas laterales y ventrales-laterales del segmento caudal V: B. turpuq n. sp. con 5-9 / 7- 


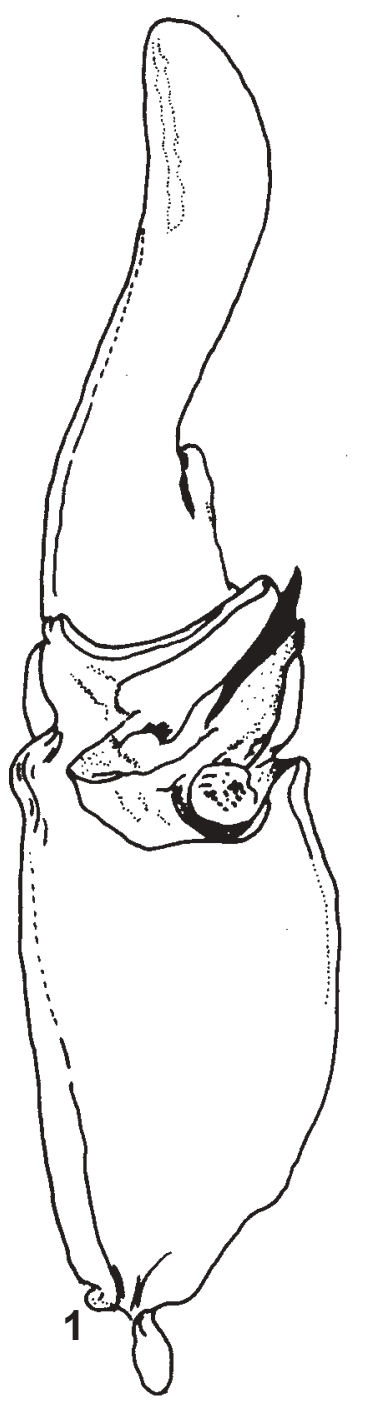

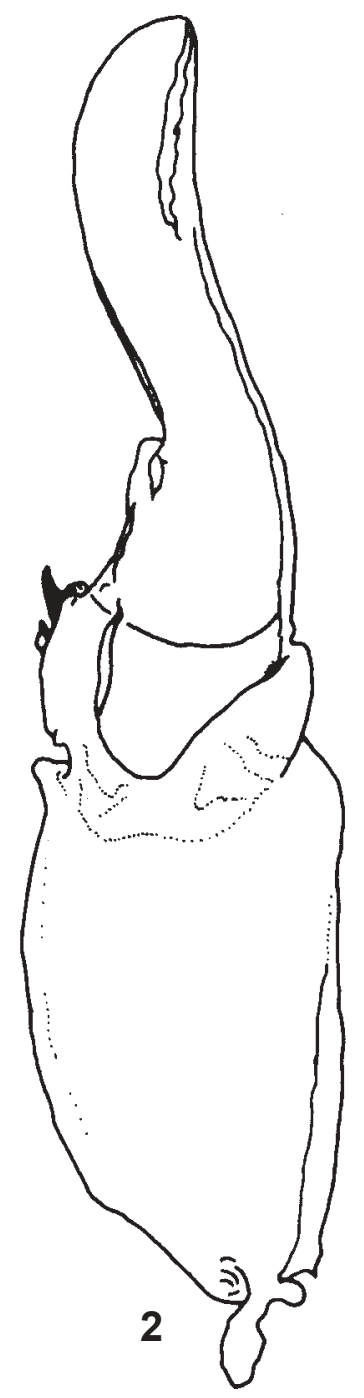

Figura 1 y 2. Brachistosternus (L.) turpuq n. sp., paratipo macho (MHNC); hemiespermatóforo izquierdo, 1, vista interna, 2, vista externa. Escala: $1 \mathrm{~mm}$.
9, B. pentheri: 9-17 / 8-13, B. multidentatus: 9-13 / 9-13, B. angustimanus: 9-15 / 10-15; respectivamente (datos según: Roig Alsina y Maury, 1984; Maury, 1984; Ojanguren Affilastro y Roig Alsina, 2001). En el telotarso III, B. turpuq n. sp. también presenta menor número de setas dorsales (9-11) y lateroventrales (7-9) en relación a $B$. multidentatus (15-18 / 12-16; Maury, 1984) y $B$. angustimanus (8-13 / 11-16; Ojanguren Affilastro y Roig Alsina, 2001). Un carácter adicional para diferenciar la nueva especie de $B$. pentheri es la ausencia en esta última de la carena Vм del segmento caudal V (Maury, 1977; Fig. 3). En cuanto al hemiespermatóforo, el conjunto de espinas del lóbulo interno son muy diminutas en $B$. turpuq $\mathrm{n}$. sp. mientras en las otras especies están bien desarolladas (Maury, 1977, Fig. 9; Ojanguren Affilastro y Roig Alsina, 2001, Fig. 12). El patrón de pigmentación ofrece también algunos caracteres diagnósticos: la parte anterior del prosoma en $B$. turpuq n. sp. presenta un pigmento lleno, mientras que $B$. pentheri y $B$. angustimanus presentan una banda mediana desde la cúpula ocular hasta el borde anterior y dos bandas oscuras oblicuas dirigidas hacia los ojos laterales (Maury, 1977; Ojanguren Affilastro y Roig Alsina, 2001); además ambas especies poseen pigmento en la cara ventral del segmento caudal 
$\mathrm{V}$, mientras que en $B$. turpuq $\mathrm{n}$. sp. el metasoma no presenta pigmentos; $B$. multidentatus presenta todo el cuerpo despigmentado (Maury, 1984). Por último cabe mencionar aquí que $B$. turpuq $\mathrm{n}$. sp. está relacionada también con una especie innominada de Chile (Atacama), diferenciándose de ella por el número de setas del segmento caudal $\mathrm{V}$ y la pigmentación de los tergitos.

\section{Descripción}

Patrón de pigmentación.Color general amarillo-naranja. Prosoma con pigmento café a café-claro en la mitad anterior (Fig. 9), el manchado es más oscuro en la región ocular, la mayoría de los ejemplares presenta una delgada línea mediana sin pigmento; el área de la foseta postocular y la porción media-lateral incluyendo los surcos transversales no presentan pigmento (a lo sumo en algunos ejemplares hay leves líneas de retículo); ángulos lateroposteriores con dos pequeñas manchitas; borde posterior con una fina línea bien marcada. Tergitos I-VI con manchado café claro en casi toda su extensión (Fig. 10); el pigmento es más evidente y oscuro en las porciones paramedianas y en el borde posterior (existen pequeñas porciones sin pigmento en las regiones laterales); en la zona que corresponde a la línea mediana el pigmento es menos evidente $\mathrm{y}$ hacia el borde posterior (especialmente en los tergitos V-VI) delimita una pequeña porción despigmentada. En algunos ejemplares el borde lateral y los ángulos latero-posteriores no presentan pigmento. Tergito VII con manchado muy suave en la porción distal. Segmentos caudales sin pigmentos, sólo se aprecian en la cara dorsal vestigios de manchas en la parte mediana y el borde posterior que se confunden con la coloración del tegumento. Quelíceros sin pigmentos. Patas y pedipalpos con algunas manchas castañas en la cara prolateral.

Morfología. Borde anterior del prosoma con una ligera prominencia mediana; surco longitudinal anterior y posterior bien marcados; tegumento del prosoma granuloso (más noto- rio en el macho). Tergitos I-VI: en los machos con gránulos, especialmente en el borde posterior; en las hembras finamente granuloso, sólo se notan gránulos mayores en el borde posterior en los segmentos IV-VI. Tergito VII granuloso, más evidente en la mitad distal, donde se notan levemente 4 carenas. Esternitos lisos en las hembras, en los machos con granulitos romos que cubren toda la extensión de los esternitos I-V, sin formar carenas. Segmentos caudales I-IV: cara dorsal y lateral de los machos granulosas, especialmente la mitad distal, disminuye en intensidad hacia el segmento IV (en las hembras sólo existen algunos gránulos distales); carenas DL y Lsm completas en el macho; en las hembras las carenas DL completas pero menos granulosas, las LsM completas en el segmento I, sólo presente en la mitad distal de los segmentos II-III y con muy pocos gránulos en el tercio distal del segmento IV; las carenas LIM delimitan junto a las Lsm una concavidad en la mitad distal del segmento I, menos evidente en II, en III existen 3 ó 4 gránulos distales y la concavidad no es evidente, en IV ausentes; carenas VL y VsM ausentes; cara ventral de los machos con la superficie granulosa (hembras lisos), que en el segmento IV es menos evidente; segmento IV con abundantes setas. Segmento caudal V (Figs. 3-5, 11-12): en los machos las carenas DL completas y acompañadas de numerosos gránulos, caras laterales con algunos gránulos esparcidos; cara ventral con gránulos en todo el segmento con las carenas VL y VM completas; VSM ausentes. En las hembras el segmento caudal V presenta una granulación menor; caras laterales mayormente lisas y la cara ventral con pocos gránulos; Vм no muy marcada. Número de setas en el segmento $\mathrm{V}$ : dorsales laterales 0 1, laterales 5-9, ventrales laterales 7-9, ventrales 5-8, la mayoría (7/8 casos) dispuesta en 3 hileras, siendo las fórmulas más frecuentes 2-2-2 y 4-2-2 (ver Variabilidad). Glándulas caudales de los machos delgadas y largas (Fig. 5). Telson alto, con la superficie ventral granular (en la hembra sólo hay gránulos 

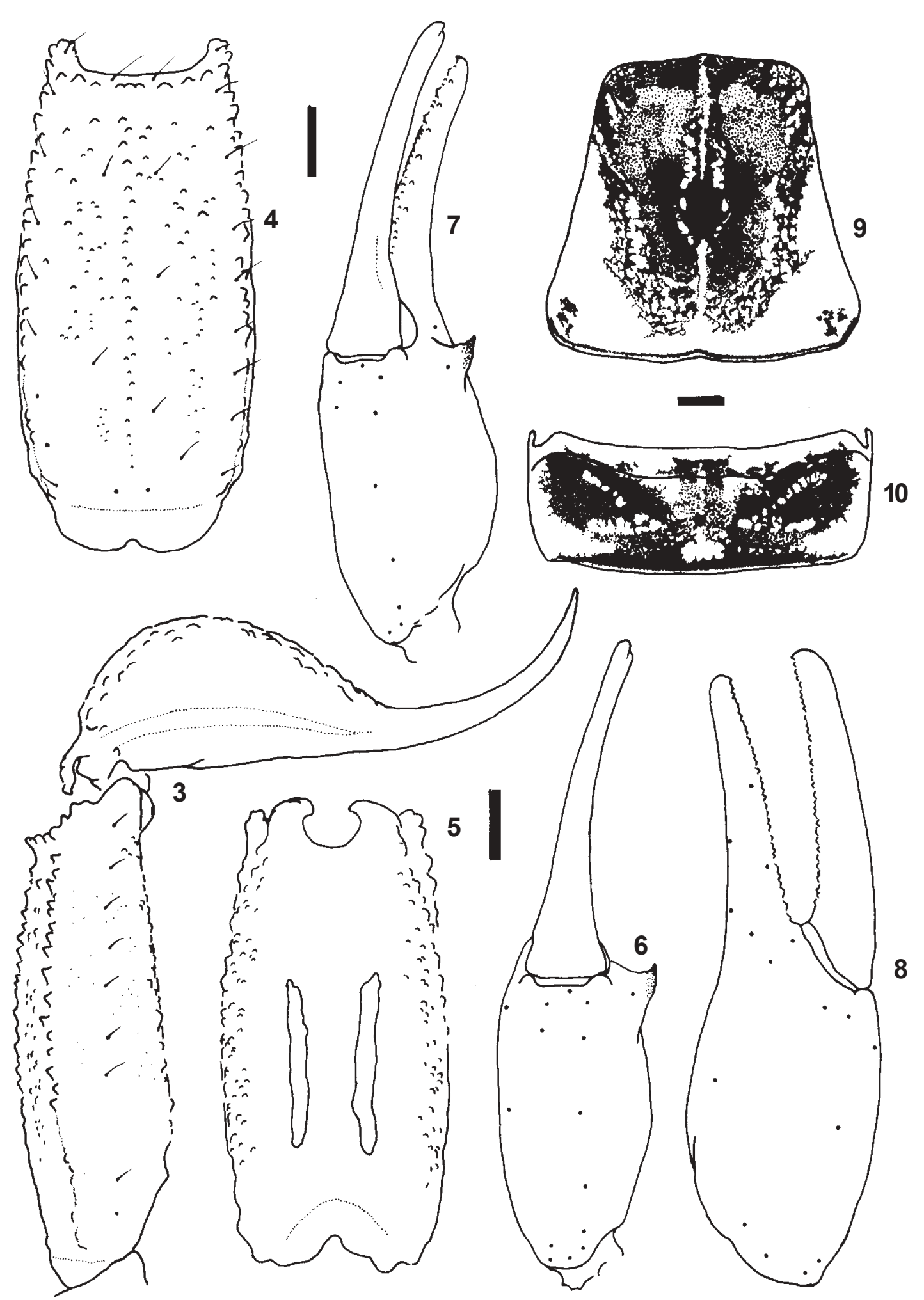

Figuras 3-10. Brachistosternus (L.) turpuq n. sp., 3-8: holotipo macho (MUSM); 3, segmento caudal $\mathrm{V}$ y telson, vista lateral; 4-5: segmento caudal $\mathrm{V}$, 4, vista ventral, 5 vista dorsal; 6-8: pinza derecha, 6, vista ventral, 7, vista ventromedial, 8, vista lateral. 9-10: macho juv. (MHNC); patrón de pigmentación, 9, prosoma, 10, tergito V. Escalas: $1 \mathrm{~mm}$. 

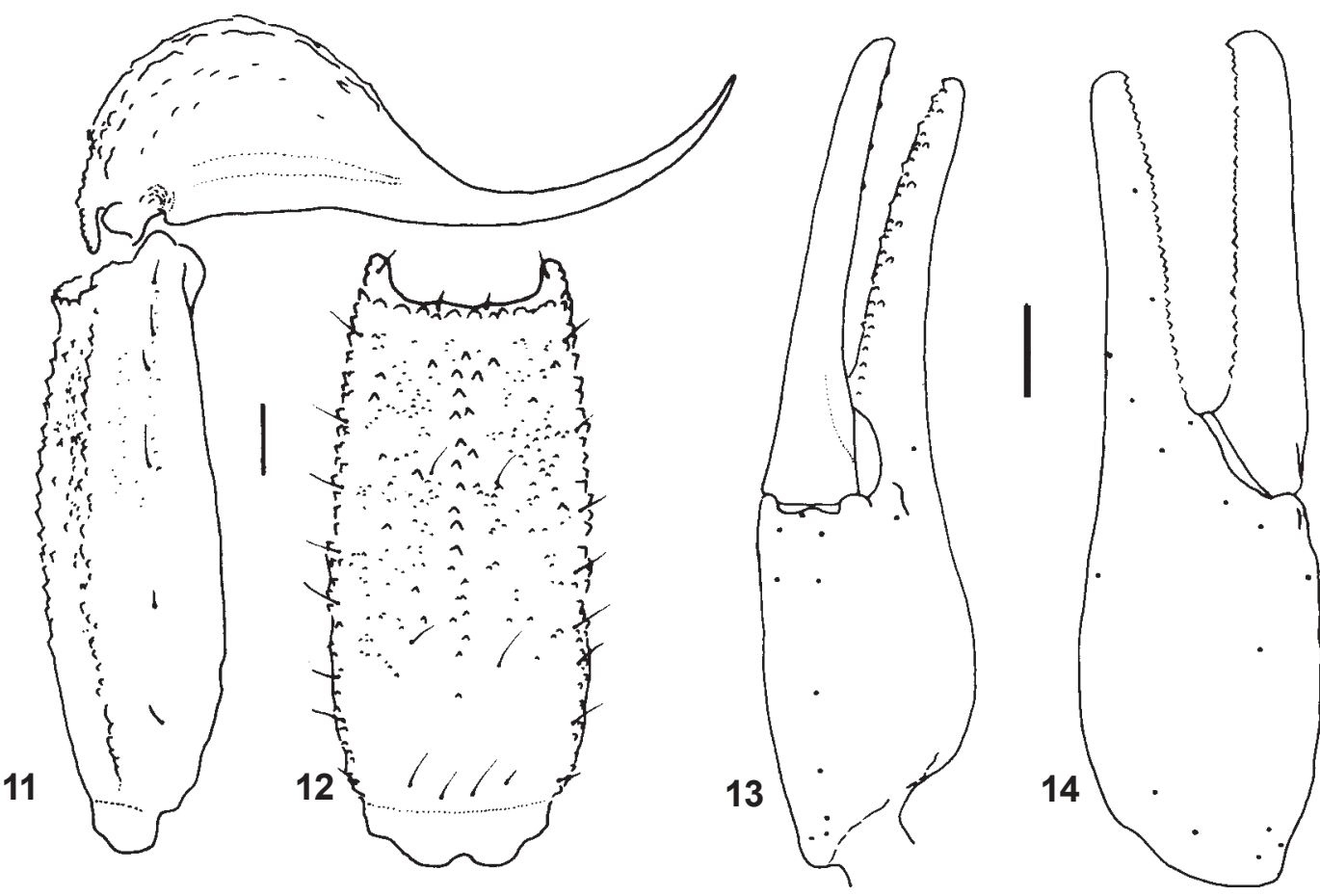

Figuras. 11-14. Brachistosternus (L.) turpuq n. sp., alotipo hembra (MUSM); 11, segmento caudal $\mathrm{V}$ y telson, vista lateral; 12, segmento caudal $\mathrm{V}$, vista ventral; 13-14: pinza derecha, 13, vista ventromedial, 14, vista lateral. Escalas: $1 \mathrm{~mm}$.

proximales); aguijón largo (Figs. 3, 11). Quelíceros con dos dientes subdistales en el dedo móvil. Pedipalpos: fémur alargado; pinza delgada con los dedos proporcionalmente largos, machos con apófisis espiniforme en la cara prolateral y la carena ventral-mediana marcada por un levantamiento del tegumento (Figs. 6-8, 13-14); dedo móvil con 6-8 dentículos internos y 6-8 externos. Tricobotriotaxia como la del subgénero (Maury, 1973). Patas granulosas en el fémur (cara prolateral), más evidente en el macho. Número de setas en el Tarso III: dorsales del telotarso 9-11, lateroventrales del telotarso 79 y dorsales del basitarso 7-9; ver Variabilidad. Número de dientes pectíneos: machos 40-42, hembras 30-33 (holotipo 41-42, alotipo 31-30; ver Variabilidad). Hemiespermatóforo (Figs. 1-2; terminología según Maury, 1975): lámina distal alargada y curvada; lóbulo distal ligeramente alargado; apófisis cilíndrica alar- gada y levemente ensanchada en el tercio distal, sobrepasa en altura a la apófisis laminar y al lóbulo interno; conjunto de espinas del lóbulo interno muy diminutas (apenas evidente); triángulo basal con numerosas espinas bien desarrolladas; conjunto de espinas del lóbulo basal no muy desarrolladas, espinas en hilera un poco más grandes.

\section{Variabilidad}

Longitud total: machos 47,2-49,9 mm $(\mathrm{n}=2)$, hembras hasta 59,8 mm. Medidas de holotipo y alotipo en Tabla 1.

Frecuencia del número de dientes pectíneos: machos ( $\mathrm{n}=6$ pectenes), 40 dientes (2 pectenes), 41 (1), 42 (3); hembras ( $\mathrm{n}=$ 10), 30 (3), 31 (3), 32 (3), 33 (1).

\section{Índice largo / ancho del segmento} caudal V: machos, 1,8-1,9 $(\mathrm{n}=2)$, holotipo: 1,8; hembras, 1,9-2,0 $(X=1,9 ; n=5)$, alotipo: 2,0. 
- Alto relativo del telson: índice largo / alto: machos, 3,5 $(\mathrm{n}=2)$ holotipo 3,5; hembras, 3,6-3,9 $(X=3,7 ; n=5)$, alotipo: 3,6.

- Índice largo / ancho del fémur del pedipalpo: machos, 3,3-3,7 $(\mathrm{n}=2)$, holotipo: 3,3; hembras, 2,8-3,2 $(X=3,0 ; n=5)$, alotipo: 3,0 .

- Índice largo / ancho de la pinza: machos, 4,2 ( $\mathrm{n}=2)$; hembras, 4,5-5,1 $(\mathrm{X}=4,7 \mathrm{n}$ $=5$ ), alotipo: 4,5 .

\section{- Frecuencia de setas del segmento caudal V:}

-Dorsales laterales $(\mathrm{n}=16)$ : 0 setas $(15$ casos), 1 (1).

—Laterales (n= 16): 5 (1), 6 (4), 7 (7), 8 (3), 9 (1).

—Ventrales laterales $(\mathrm{n}=16): 7(1), 8(13)$, 9 (2).
—Ventrales (n=8): 5 (1), $6(3), 8$ (4).

- Frecuencia de setas en el Tarso III $(n=16)$ :

—Dorsales del telotarso: 9 (4), 10 (5), 11 (7).

-Lateroventrales del telotarso: 7 (2), 8 (13), 9 (1).

—Dorsales del basitarso: 7 (6), 8 (9), 9 (1).

\section{Distribución y hábitat}

Sur del Perú, departamento de Arequipa (Fig. 15). Por el momento sólo se ha encontrado esta especie en los cerros presentes en los alrededores de la localidad de Mejía (provincia de Islay) a 340 metros de altitud. La zona corresponde a la cadena montañosa de la costa que está presente en el sur del Perú y corre paralela al litoral Pacífico, pertenecien-

Tabla 1. Medidas ( $\mathrm{mm}$ ) del holotipo macho y el alotipo hembra de Brachistosternus turpuq n. sp.

\begin{tabular}{|c|c|c|}
\hline & Holotipo & Alotipo \\
\hline Longitud total & 49,9 & 59,8 \\
\hline Prosoma, largo & 5,7 & 7,5 \\
\hline ancho anterior / posterior & $3,9 / 6,9$ & $4,7 / 7,9$ \\
\hline Mesosoma, largo & 12,2 & 15,3 \\
\hline Metasoma, largo & 32,0 & 37,0 \\
\hline Segmento caudal I, largo / ancho & $3,7 / 3,6$ & $4,3 / 4,3$ \\
\hline Segmento caudal II, largo / ancho & $4,4 / 3,2$ & $5,0 / 3,9$ \\
\hline Segmento caudal III, largo / ancho & $4,7 / 3,1$ & $5,4 / 3,7$ \\
\hline Segmento caudal IV, largo / ancho & $5,5 / 3,1$ & $6,1 / 3,5$ \\
\hline Segmento caudal V, largo / ancho / alto & $6,0 / 3,3 / 2,2$ & $7,1 / 3,5 / 2,4$ \\
\hline Telson, largo & 7,7 & 9,1 \\
\hline Vesícula, largo / ancho / alto & $4,4 / 2,5 / 2,2$ & $4,8 / 2,7 / 2,5$ \\
\hline Aguijón, largo & 3,3 & 4,3 \\
\hline Pedipalpo, largo total & 17,7 & 18,1 \\
\hline Fémur, largo / ancho & $4,6 / 1,4$ & $4,5 / 1,5$ \\
\hline Patela, largo / ancho & $4,3 / 1,7$ & $4,5 / 1,9$ \\
\hline Pinza, largo / ancho / alto & $8,8 / 2,1 / 2,7$ & $9,1 / 2,0 / 2,5$ \\
\hline Dedo móvil largo & 4,9 & 5,3 \\
\hline
\end{tabular}




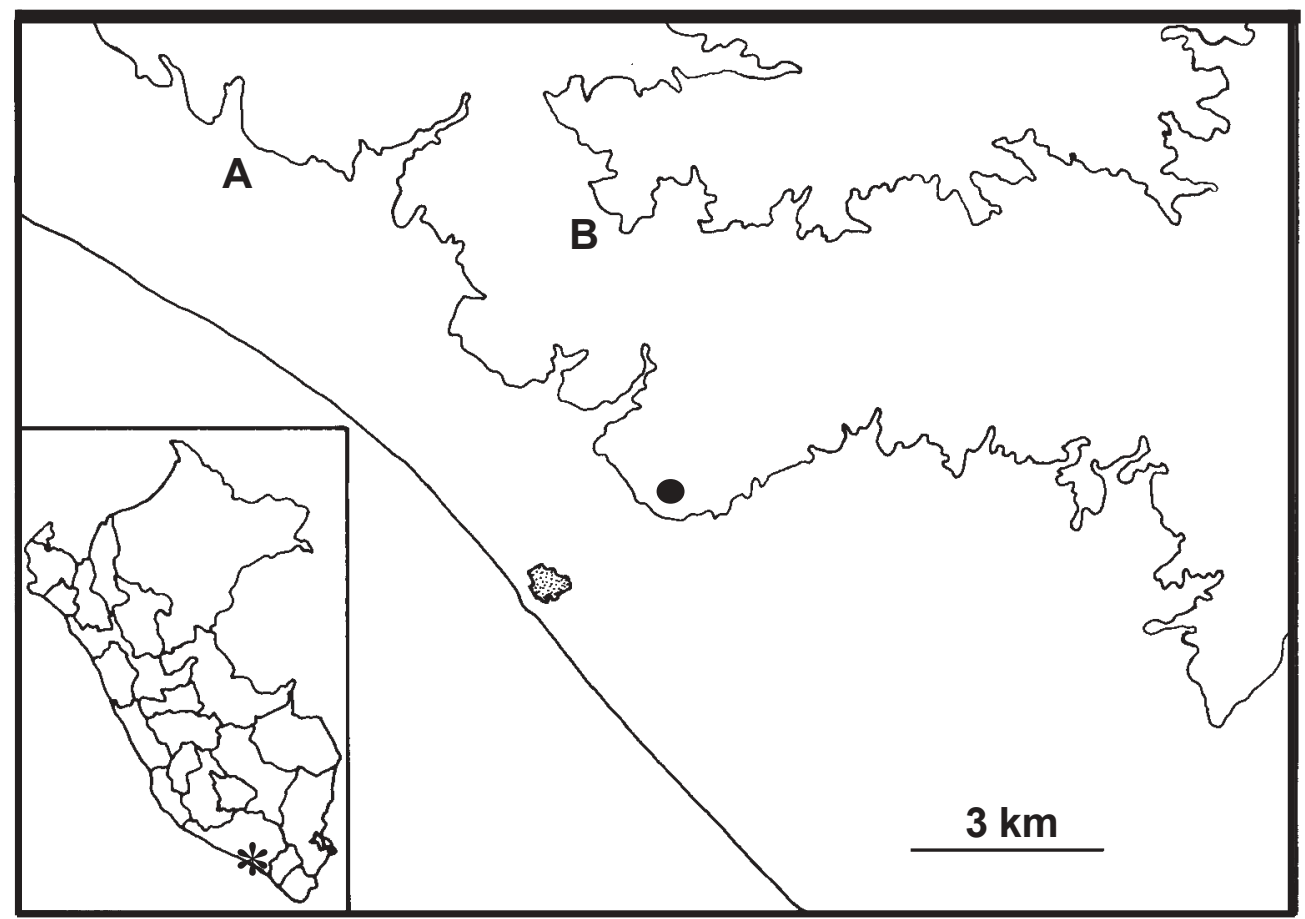

Figura. 15. Localidad estudiada de Brachistosternus (L.) turpuq n. sp., en el Departamento de Arequipa. Con un círculo negro se señala la ubicación del lugar de muestreo. Cotas de altitud: A, 200 m, B, 600 m. Me = Localidad de Mejía. Recuadro inferior izquierdo: ubicación en el Perú del área representada (asterisco pequeño).

do a la denominada Ecorregión del Desierto Pacífico (Brack, 1986). En el lugar de captura el suelo es árido-seco, de color rojizo, ligeramente compacto y por sectores de consistencia algo granuloso; la pendiente es suave y no hay piedras. La vegetación es muy escasa, salvo algunos arbustos, cactáceas y gramíneas. El área se encuentra inmediatamente por encima de la zona arenosa que caracteriza al litoral Pacífico y correspondería a los inicios de las laderas donde metros más arriba se forma la vegetación de las «lomas» (Péfaur, 1981). Todos los ejemplares fueron capturados durante la noche con luz UV.

\section{Agradecimientos}

Agradezco al Blgo. Horacio Zeballos P., Henrry Alayo, Ulrich Zanabria y Alejandro Pari (Universidad Nacional de San Agustín de Arequipa) por la ayuda en las tareas de co- lecta y por la información geográfica del área de estudio. Al Ing. Marco Arenas y al Blgo. Arturo Cornejo por todas las facilidades prestadas y el uso de las instalaciones de la Reserva Nacional de las Lagunas de Mejía durante mi permanencia en dicho lugar. Al Blgo. Camilo Mattoni por sus comentarios al manuscrito. El presente trabajo forma parte de mi tesis doctoral realizado en la Facultad de Ciencias Exactas, Físicas y Naturales de la Universidad Nacional de Córdoba, Argentina, bajo la dirección del Dr. Luis E. Acosta, a quien quedo muy agradecido.

\section{Literatura citada}

Acosta, L. E. y E. A. Maury. 1998. Scorpiones. En: Morrone, J. J. \& Coscarón, S. (Eds.): Biodiversidad de Artrópodos argentinos. Una perspectiva biotaxonómica. Ed. Sur, La Plata. pp. 545-559. 
Aguilar, P. G. y O. Meneses. 1970. Escorpiones y escorpionismo en el Perú. I. Nota preliminar sobre los Scorpionida peruanos. Anales Científicos de la Universidad Nacional Agraria, 8 (1-2):1-5.

Brack, A. 1986. Ecología de un país complejo. In: Gran Geografía del Perú. Naturaleza y Hombre. Manfer-Mejía Baca, Lima, t. 2, pp. 175-319.

Francke, O. F. 1977. Escorpiones y Escorpionismo en el Perú VI: Lista de especies y claves para identificar las familias y los géneros. Revista Peruana de Entomología, 20: 73-76.

International Commission on Zoological Nomenclature. 1999. International Code of Zoological Nomenclature, Fourth edition, London: International Trust for Zoological Nomenclature.

Lowe, G. y V. Fet. 2000. Family Bothriuridae Simon, 1880. En: Fet, V., W. D. Sissom, G. Lowe y M. E. Braunwalder (Eds.). Catalog of the Scorpions of the World (1758-1998). The New York Entomological Society, New York, pp. 17-53.

Maury, E. A. 1973. Las tricobotrias y su importancia en la sistemática del género Brachistosternus Pocock 1894 (Scorpiones, Bothriuridae). Physis, secc. C, 32 (85): 247-254.

Maury, E. A. 1975. La estructura del espermatóforo en el género Brachistosternus (Scorpiones, Bothriuridae). Physis, secc. C, 34 (89): 179-182.
Maury, E. A. 1977. Un nuevo Brachistosternus de los médanos costeros bonaerenses (Scorpiones, Bothriuridae. Physis, secc. C, 37 (93): 169-176.

Maury, E. A. 1978. Escorpiones y escorpionismo en el Perú VII. Nuevos hallazgos y redescripción de Brachistosternus (Microsternus) andinus Chamberlin, 1916 (Bothriuridae). Revista Peruana de Entomología, 21 (2): 23-26.

Maury, E. A. 1984. Una nueva especie de Brachistosternus de la Argentina (Scorpiones, Bothriuridae). Revista de la Sociedad Entomológica Argentina, 43 (1-4): 113-118.

Ochoa, J. A. y L. E. Acosta. 2002. Two New Andean Species of Brachistosternus Pocock (Scorpiones: Bothriuridae). Euscorpius, Occasional Publications in Scorpiology, 2: 1-13.

Ojanguren Affilastro, A. A. y A. Roig Alsina. 2001. Brachistosternus angustimanus, una nueva especie del norte de la Patagonia, Argentina (Scorpiones, Bothriuridae). Physis, secc. C, 58 (134-135):15-22.

Péfaur, J. E. 1981. Composition and phenology of epigeic animal communities in the Lomas of southern Peru. Journal of Arid Environments, 4: 31-42.

Roig Alsina, A. y E. A. Maury. 1984. Sistemática y distribución geográfica de Brachistosternus (L.) pentheri Mello-Leitão, 1931 (Scorpiones, Bothriuridae). Una nueva especie de escorpión andino de Mendoza, República Argentina (Bothriuridae). Physis, secc. C, 42 (102): 17-21. 